\title{
Caracterização Biológica e Fisiológica de Isolados de Sclerotium rolfsii Obtidos de Pimentão no Estado do Maranhão*
}

\author{
Ilka Márcia R. de S. Serra ${ }^{1 * *}$ \& Gilson S. da Silva ${ }^{2}$ \\ ${ }^{1}$ Departamento de Agronomia e Fitossanidade, Universidade Federal Rural de Pernambuco, Dois Irmãos, CEP 52171-900, \\ Recife, PE, e-mail: ilka.tt@terra.com.br; ${ }^{2}$ Departamento de Fitotecnia e Fitossanidade, Universidade Estadual do Maranhão, \\ CEP 65001-970, São Luís, MA
}

(Aceito para publicação em 01/10/2004)

Autor para correspondência: Ilka Márcia R. de S. Serra

SERRA, I.M.R.S. \& SILVA, G.S. Caracterização biológica e fisiológica de isolados de Sclerotium rolfsii obtidos de pimentão no estado do Maranhão. Fitopatologia Brasileira 30:61-66. 2005.

\section{RESUMO}

A murcha de esclerócio do pimentão (Capsicum annum), causada por Sclerotium rolfsii é uma das mais importantes doenças dessa solanácea, nas regiões tropicais. Um dos requisitos básicos para obtenção de sucesso no controle dessa doença é o conhecimento do patógeno, especialmente de fatores relacionados à fisiologia e biologia. O presente trabalho teve como objetivo caracterizar cinco isolados de S. rolfsii, obtidos de pimentão, quanto ao crescimento micelial, produção de escleródios em diferentes regimes de luminosidade e em seis substratos naturais e a compatibilidade vegetativa entre os isolados. O efeito do substrato variou com o isolado na produção de escleródios. Os isolados SR3 e SR5 apresentaram maior produção de escleródios nos substratos aveia-ágar (AA) e fubá-ágar (FA), SR1 em casca de arroz (CA), SR2 em AA e SR4 em FA. O substrato CA favoreceu a formação de escleródios na maioria dos isolados. Quanto à taxa de crescimento micelial os isolados apresentaram pouca variação, sendo SR1, SR2 e SR3 os que apresentaram maior crescimento. Os cincos isolados exibiram comportamento diferente quando submetidos a três regimes de luz (luz contínua, alternância luminosa e escuro contínuo), tendo o primeiro favorecido maior produção de escleródios em relação aos outros. O teste de compatibilidade vegetativa mostrou diversidade genética entre os cincos isolados, possibilitando a classificação em três grupos. Os isolados SR3 e SR5 foram incompatíveis com os demais e entre si. Houve compatibilidade vegetativa entre os isolados SR1, SR2 e SR4. De acordo com os aspectos culturais e fisiológicos estudados, os isolados de S. rolfsii SR3 e SR5 podem ser considerados "strains" diferentes, enquanto SR1, SR2 e SR4 podem ser considerados da mesma "strain".

Palavras-chave adicionais: Capsicum annum, Sclerotium sp., compatibilidade vegetativa, produção de escleródios.

\begin{abstract}
Biological and physiological characterization of Sclerotium rolfsii isolates, obtained from green pepper in the State of Maranhão Green pepper (Capsicum annum) sclerotium wilt, caused by Sclerotium rolfsii, is one of the most important diseases of this solanaceous in tropical regions. One of the basic requirements for successful in controll of this disease is knowledge of the pathogen, especially of factors related to physiology and biology. The present work aimed to characterize five isolates of $S$. rolfsii (SR1, SR2, SR3, SR4 e SR5), from green pepper, accessing the mycelial growth, sclerotia production in different photophases and in six natural substracts and the vegetative compatibility among these isolates. The substract effect varied within isolates for sclerotia production. The SR3 and SR5 presented the highest sclerotium production in oat (Avena sativa)-agar (OA) and flour-agar (CA), SR1 in rice (Oryza sativa) hull (RH), SR2 in OA and SR4 in CA. The RH favored sclerotia formation in most isolates. For the mycelial growth rate, the isolates tested showed low variation, but SR1, SR2 and SR3 presented higher growth. The five isolates showed differential behavior when submitted to three different photophases: continuous light (CL), alternating light and dark (L/D) and continuous dark (CD), the highest sclerotia production was found under CL. The vegetative compatibility test showed genetic diversity among the five isolates, clustering them into three groups. The SR3 and SR5 were incompatible one to other and with all other isolates. There was vegetative compatibility among isolates SR1, SR2 and SR4. According to the biological and physiological aspects studied the isolates of S. rolfsii SR3 and SR5 can be considered different "strains", while SR1, SR2 and SR4 belong to the same strain.

Additional keywords: Capsicum annum, Sclerotium sp., sclerotia production, vegetative compatibiliy.
\end{abstract}

\section{INTRODUÇÃO}

O pimentão (capsicum annum L.) é uma hortaliça tipicamente de clima tropical. No Brasil é cultivado em todo território nacional, sendo uma das dez hortaliças de maior

\footnotetext{
*Trabalho de conclusão do curso de Agronomia da primeira autora. Universidade Estadual do Maranhão (2001).

**Bolsista do CNPq
}

Fitopatol. bras. 30(1), jan - fev 2005 importância econômica no mercado hortigranjeiro brasileiro, podendo ser consumida sob a forma de frutos verdes, maduros e mesmo industrializados, em forma de pó. No Nordeste, destacam-se como principais produtores os Estados de Pernambuco, Paraíba, Ceará e Bahia em ordem decrescente (Ceasa-PE, 1992).

Dentre as doenças que afetam o pimentão, a murcha de esclerócio, causada por Sclerotium rolfsii Sacc. é uma das 
mais relevantes. O fungo é um importante fitopatógeno habitante de solo, sendo responsável por podridão de raízes e do colo, murcha e tombamento de plântulas. Apresenta extensa gama de hospedeiros, cerca de 500 espécies botânicas, incluindo dicotiledôneas e monocotiledôneas, distribuindose em todas as regiões agrícolas, com predominância nas zonas tropical e subtropical, onde predominam condições de alta umidade e temperatura elevada (Aycock, 1966; Punja \& Jenkins, 1984; Punja, 1985; Punja \& Rahe, 1992).

Desde o primeiro trabalho de Rolfs em 1892, com $S$. rolfsii na cultura do tomateiro (Lycopersicon esculentum Mill.), muitos trabalhos foram desenvolvidos sobre o fungo e, apesar disso, esse patógeno continua a causar problemas em diferentes culturas, trazendo prejuízos para a produção (Epps et al., 1951). No Estado do Maranhão, essa doença tem ampla distribuição geográfica e causa perdas elevadas, chegando a 100\% em algumas áreas onde se cultiva o pimentão.

Sabe-se que isolados de $S$. rolfsii originados de diferentes áreas geográficas e hospedeiros apresentam variação nas características morfológicas e físiológicas (Punja \& Grogan, 1983). Diferenças entre espécies são facilmente identificadas pelo tamanho, cor e forma de escleródios (Punja \& Rahe, 1992). Entretanto, segundo Harlton et al. (1995), não está devidamente esclarecido que quaisquer dessas diferenças sejam suficientes para garantir a separação de isolados dentro da espécie $S$. rolfsii. Uma das técnicas que pode ser empregada nesse estudo é a da compatibilidade vegetativa entre isolados, que tem sido usada para avaliar a variabilidade genética em diversos fungos patogênicos à plantas (Leslie, 1993; Dantas, 1999). Nakata (1925) separou isolados de $S$. rolfsii com base na formação de zona de aversão, ou ausência desta, quando os isolados foram pareados em meios de cultura, sendo os mesmos reunidos em grupos de compatibilidade vegetativa (GCV). Desde então a GCV vem sendo usada na caracterização de isolados de $S$. rolfsii.

Considerando os fatores físicos que exercem efeito no crescimento e formação esclerodial, destaca-se a luz. Segundo Punja (1985), há melhor desenvolvimento de S. rolfsii, em condições de luminosidade continua, sugerindo que a produção abundante de escleródios na superfície do solo seja em resposta à presença de luz. Essa observação foi também relatada por outros pesquisadores, como McClellan et al., (1955) que verificaram maior produção de escleródios em culturas expostas a luz.

Diversos meios são utilizados para produção de escleródios de $S$. rolfsii, segundo Pérez Sendín et al. (1986) o fungo apresenta bom crescimento micelial e formação de escleródios em meios de farinha de milho e extrato de malte. Punja (1985) observou que $S$. rolfsii possui grande adaptabilidade a diferentes fontes de carbono e também utiliza numerosos compostos orgânicos e inorgânicos como fontes de nitrogênio. Segundo observações de Henis et al. (1965) e Zoberi (1980), a glucose é uma importante fonte de carbono para o crescimento micelial e a produção de escleródios.
O controle dessa doença é difícil, em razão da enorme gama de hospedeiros apresentada pelo fungo, bem como pela longa sobrevivência do patógeno no solo. Na definição de estratégias de controle, é fundamental conhecer aspectos da biologia e fisiologia do patógeno, destacando-se o desenvolvimento de meios de cultura onde o patógeno-alvo se reproduza abundantemente. A busca de meios de culturas de baixo custo e eficientes para induzir a produção de escleródios é de grande importância para estudos do patógeno.

O presente trabalho teve como objetivos caracterizar isolados de $S$. rolfsii patogênicos ao pimentão, avaliando o seu crescimento micelial, e a compatibilidade vegetativa entre diferentes isolados; o efeito de diferentes substratos e a influência da luminosidade na produção de escleródios.

\section{MATERIALEMÉTODOS}

Utilizaram-se cinco isolados do fungo, obtidos de pimentão, cultivados em diferentes localidades no Maranhão. Os isolados SR1 e SR5 foram oriundos de Paço do Lumiar, SR3 de Buriti, e os isolados SR2 e SR4 procedentes de São Luís. O fungo foi isolado em meio de cultura batata-dextroseágar (BDA) e, posteriormente, incubado em estufa do tipo Biochemistry Oxygen Demand (B.O.D.) à temperatura de 25 ${ }^{\circ} \mathrm{C}$, com fotoperíodo de $12 \mathrm{~h}$.

Para o estudo das características fisiológicas, discos de micélio e meio de cultura (5,0 mm de diâmetro), oriundos de colônia jovem de cada isolado, foram transferidos para o centro de placa de Petri, contendo meio BDA, as quais foram incubadas a $25^{\circ} \mathrm{C}$, com $12 \mathrm{~h}$ de fotoperíodo. Foi observado o crescimento do micélio, medindo-se o diâmetro da colônia em intervalos de 24 h, com auxílio de uma régua milimetrada, tomando-se duas medições, em sentidos diametralmente opostos. Em seguida, foi estabelecida a média de crescimento linear para cada placa. As leituras foram iniciadas $24 \mathrm{~h}$ após a instalação do experimento, até a colonização de toda a superfície do meio de cultura, que correspondeu a três dias de incubação. O cálculo da velocidade de crescimento foi realizado de acordo com Lilly \& Barnett (1951).

O delineamento experimental utilizado foi o inteiramente casualizado com quatro repetições, sendo cada repetição constituída por uma placa de Petri. As médias dos dados obtidos foram comparadas utilizando-se o teste de Tukey ao nível de 5\% de probabilidade.

\section{Compatibilidade vegetativa entre os isolados de $S$. rolfsii}

A compatibilidade vegetativa entre os isolados foi avaliada em placas de Petri de $9 \mathrm{~cm}$ de diâmetro. Um escleródio de cada isolado foi transferido para placas contendo BDA e pareados em posições opostas e eqüidistantes um do outro. A incubação foi feita à temperatura de $25 \pm 1^{\circ} \mathrm{C}$, em regime de luz alternada com fotoperíodo de $12 \mathrm{~h}$. Foram utilizadas quatro repetições. Após a incubação durante três dias, as placas foram avaliadas macroscopicamente observando-se a presença ou não de antagonismo (zona de aversão) na região de contato micelial. 
Caracterização biológica e fisiológica de isolados de Sclerotium rolfsii...

Efeito da luminosidade na capacidade de produção de escleródios dos isolados de $S$. rolfsii

O meio BDA foi usado para realização desse experimento, por induzir bom crescimento micelial, abundante formação de escleródios, facilitando a contagem dos mesmos, devido à transparência do meio.

Discos de cultura jovem de $S$. rolfsii, com $5 \mathrm{~mm}$ de diâmetro, foram repicados para o centro de placas de Petri contendo BDA, e incubadas sob três regimes de luminosidade: luz contínua, escuro contínuo e alternância luminosa ( $12 \mathrm{~h}$ de luz e $12 \mathrm{~h}$ escuro), à temperatura de $25 \pm 1{ }^{\circ} \mathrm{C}$. Vinte dias após, as culturas foram avaliadas quanto à produção de escleródios. Procedeu-se a remoção dos escleródios com auxílio de uma pinça e a contagem de todos os escleródios produzidos para cada isolado e tratamento. O delineamento estatístico utilizado foi o inteiramente casualizado, constando de 15 tratamentos, representados pelos três regimes de luz e os cincos isolados, com quatro repetições.

\section{Produção de escleródios em seis diferentes substratos}

Para avaliar a produção de escleródios os substratos utilizados foram: a) grãos de arroz (Oryza sativa L.) beneficiados (AB); b) sementes de sorgo [Sorghum bicolor (L.) Moench] (SS); c) casca de arroz (CA); d) quirera de milho (Zea mays L.)-ágar (QA); e) fubá-ágar (FA); f) aveia (Avena sativa L.)-ágar (AA).

Para os tratamentos AB e SS foram utilizados $20 \mathrm{~g}$ de cada substrato, e para a CA foram usados $5 \mathrm{~g}$. Os substratos foram embebidos em água destilada por 10 min e autoclavados por $30 \mathrm{~min}$, em dois dias consecutivos. Os meios de QA, FA, AA foram preparados usando a metodologia de Menezes \& Silva-Hanlin (1997), onde se utilizou 75,0 g de cada substrato; $17,0 \mathrm{~g}$ de ágar e água destilada em quantidade suficiente para $1.000 \mathrm{ml}$.

Em cada substrato, foram depositados discos de micélio e meio de cultura (5,0 $\mathrm{mm}$ de diâmetro), oriundos de colônias jovens de cada isolado de $S$. rolfsii, desenvolvidos em BDA. Após a inoculação, os substratos foram incubados durante 25 dias em câmara do tipo B.O.D. a $25 \pm 2{ }^{\circ} \mathrm{C}$, com fotoperíodo de $12 \mathrm{~h}$. Ao término da incubação procedeu-se a remoção dos escleródios conforme citado no experimento anterior.

Foi utilizado um delineamento experimental inteiramente casualizado, em esquema fatorial $5 \times 6$, constando de 30 tratamentos, representados pelos cincos isolados de $S$. rolfsii e os seis diferentes substratos, com quatro repetições. As médias dos dados obtidos foram comparadas utilizandose o teste de Tukey ao nível de $5 \%$ de probabilidade, sendo os dados transformados em $\sqrt{ } \mathrm{x}+0,5$.

Todas as análises estatísticas foram realizadas utilizando-se o SANEST (Sistema para análise estatística).

\section{RESULTADOS E DISCUSSÃO}

Os resultados obtidos para taxa de crescimento micelial dos cinco isolados de $S$. rolfsii são apresentados na Tabela 1.
Embora tenha ocorrido pouca variação entre os isolados, foi possível separá-los em dois grupos distintos. O isolado SR5 foi o que apresentou menor velocidade de crescimento, diferindo $(\mathrm{P} \leq 0,05)$ dos demais, exceto de SR4.

As diferenças nas taxas de crescimento micelial verificadas podem ser atribuídas à variabilidade genética entre os isolados, fato que pode ser observado nos outros aspectos estudados no presente trabalho.

Os resultados observados nesse experimento diferem daqueles obtidos por Lang (1989), que encontrou para $S$. rolfsii, em meio BDA, uma taxa de crescimento micelial entre 150 a $300 \mu \mathrm{m} / \mathrm{h}$, a $25^{\circ} \mathrm{C}$ e por Punja (1985), o qual verificou taxas médias de 850 a $970 \mu \mathrm{m} / \mathrm{h}$, a $27{ }^{\circ} \mathrm{C}$. Estes dados, visivelmente superiores aos encontrados neste trabalho, podem ser indicativo da variabilidade entre os isolados $S$. rolfsii usados nestes e nos outros trabalhos mencionados.

\section{Compatibilidade vegetativa entre os isolados de $S$. rolfsii}

Os resultados referentes à compatibilidade vegetativa mostraram que as hifas dos isolados SR1 (proveniente de Paço do Lumiar) e os isolados SR2 e SR4 (provenientes de São Luis) formaram anastomose, ou seja, houve superposição de uma colônia sobre a outra, conforme mostra a Figura 1A. Isto indica que esses isolados podem ser agrupados em um mesmo GCV (grupo compatível vegetativamente). Alguns autores consideram que, nesse caso, há similaridade genética entre os isolados e uma provável origem geográfica comum, tendo também, semelhantes atributos biológicos, fisiológicos e patogênicos (Punja \& Grogan, 1983; Katan et al., 1991; Harlton et al. 1995). Não foi observada anastomose de hifas nos isolados SR3 (proveniente de Buriti) e SR5 (proveniente de Paço do Lumiar), quando pareados com os outros isolados, ocorrendo uma zona de aversão entre os mesmos, indicando incompatibilidade (Figura 1B) e, provavelmente dissimilaridade genética. No pareamento dos isolados SR3 e SR5, foi observada a ocorrência de zona de aversão, sugerindo que os mesmos não apresentam compatibilidade vegetativa, logo pertencem a GCV diferentes.

\section{Efeito da luminosidade na capacidade de produção de escleródios dos isolados de $S$. rolfsii}

Os resultados obtidos para influência da luminosidade na produção de escleródios dos cinco isolados de $S$. rolfsii (Tabela 2) mostraram interação entre os isolados e regime de luminosidade. Os isolados SR3 e SR5 produziram maior $(\mathrm{P} \leq 0,05)$ número de escleródios sob luz contínua e menor sob escuro contínuo. Resultados semelhantes foram obtidos por Pérez Sendín et al. (1986) e McClellan et al. (1955) os quais conseguiram maior número de escleródios do fungo em condições de claro contínuo e menor quantidade de escleródios em escuro contínuo.

Em condições de luz contínua e alternância luminosa, os isolados SR3 e SR5 superaram os demais $(\mathrm{P} \leq 0,05)$, concordando com os resultados obtidos por Lang (1989) e Blum et al. (1993) que verificaram efeito da luz na produção de escleródios de diferentes isolados de $S$. rolfsii, principalmente 
TABELA 1 - Taxa de crescimento micelial de cinco isolados de Sclerotium rolfsii em meio BDA a temperatura de $25 \pm 1{ }^{\circ} \mathrm{C}$

\begin{tabular}{lc}
\hline \hline $\begin{array}{l}\text { Isolado de } \\
\text { Sclerotium rolfssi }\end{array}$ & $\begin{array}{c}\text { Taxa de Crescimento Micelial } \\
(\mu \mathbf{m} / \mathbf{h o r a}) *\end{array}$ \\
\hline SR3 & $152,2 \mathrm{a}$ \\
SR2 & $149,5 \mathrm{a}$ \\
$\mathrm{SR} 1$ & $146,5 \mathrm{a}$ \\
$\mathrm{SR} 4$ & $126,8 \mathrm{ab}$ \\
$\mathrm{SR} 5$ & $113,5 \mathrm{~b}$ \\
\hline C.V.=6,57\% \\
* Média de quatro repetições. Médias seguidas da mesma letra, não \\
diferem entre si ao nível de 5\% de probabilidade, pelo teste de Tukey.
\end{tabular}

quando os isolados foram submetidos às condições de claro contínuo e alternância luminosa. Entretanto, em condição de escuro contínuo, embora os isolados SR3 e SR5 tenham diferido em relação aos demais isolados, SR3 foi o que apresentou maior $(\mathrm{P} \leq 0,05)$ produção de escleródios (Tabela 2$)$.

Não houve efeito dos regimes de luz utilizados sobre a produção de escleródios pelos isolados SR1, SR2 e SR4 constatando-se que há variação entre os isolados quanto a este critério.

\section{Produção de escleródios em seis diferentes substratos}

Os resultados obtidos para a produção de escleródios dos cinco isolados de $S$. rolfsii, nos diferentes substratos utilizados no experimento (Tabela 3) mostraram que o efeito do substrato variou com o isolado. O isolado SR1 produziu maior $(\mathrm{P} \leq 0,05)$ número de escleródios em casca de arroz, $\mathrm{SR} 3$ e SR5 em aveia e fubá, SR4 em fubá e SR2 em aveia, fubá e casca de arroz. Esses resultados podem ser comparados com os obtidos por Lang (1989), que constatou uma maior produção de escleródios em meio de aveia-ágar e os obtidos por Blum et al. (1993); Fernandes et al. (1993); Ferraz \& Café Filho (1998), que verificaram maior formação de escleródios com a adição de fubá de milho aos meios de cultura.

Todos os isolados, exceto SR1, produziram escleródios em sorgo concordando com Beute \& Rodriquez-Kabana (1979) que obtiveram grande número escleródios de $S$. rolfsii em sementes de sorgo autoclavadas. Resultados interessantes com este substrato foram obtidos por Neto \& Blum (2004) que verificaram que semente de sorgo proporcionou bom crescimento micelial e germinação de escleródios de $S$. rolfsii.

De maneira geral, o arroz beneficiado não favoreceu a produção de escleródios em quaisquer dos isolados testados. No entanto, este resultado difere daqueles obtidos por Matsumoto et al. (2000), que obtiveram grande quantidade de escleródios tanto em arroz autoclavado como cru. A discordância dos resultados pode ser atribuída à variabilidade e a origem dos isolados. Matsumoto et al. (2000) utilizaram isolados obtidos de pepino e feijoeiro, procedentes do Paraná, região de clima e solo amplamente distintos do Maranhão, local de origem dos isolados estudados neste trabalho.

O substrato casca de arroz favoreceu a produção esclerodial de todos os isolados, com exceção do SR4, que somente iniciou o processo de produção após a leitura final do experimento. Não foram encontradas referências ao uso

TABELA 2 - Influência de três regimes de luz na produção de escleródios por cinco isolados de Sclerotium rolfsii, em meio BDA

\begin{tabular}{|c|c|c|c|c|c|}
\hline \multirow{3}{*}{ Luz } & \multicolumn{5}{|c|}{ Número de escleródios produzidos* } \\
\hline & \multicolumn{5}{|c|}{ Isolados de Sclerotium rolfsii } \\
\hline & SR1 & SR2 & SR3 & SR4 & SR5 \\
\hline Luz contínua & $15,75 \mathrm{aB}$ & $55,25 \mathrm{aB}$ & $969,75 \mathrm{aA}$ & $15,00 \mathrm{aB}$ & $986,00 \mathrm{aA}$ \\
\hline Alternância luminosa & $2,00 \mathrm{aB}$ & $17,25 \mathrm{aB}$ & $844,50 \mathrm{bA}$ & $3,00 \mathrm{aB}$ & $894,20 \mathrm{bA}$ \\
\hline Escuro contínuo & $0,00 \mathrm{aC}$ & $3,00 \mathrm{aC}$ & $525,50 \mathrm{cA}$ & $0,00 \mathrm{aC}$ & $399,25 \mathrm{cB}$ \\
\hline
\end{tabular}

* Média de quatro repetições. Médias seguidas pela mesma letra, minúscula no sentido vertical e maiúscula no sentido horizontal, não diferem entre si ao nível de $5 \%$ de probabilidade, pelo teste de Tukey.

TABELA 3 - Produção de escleródios por cinco isolados de Sclerotium rolfsii, em seis substratos, após 25 dias de incubação

\begin{tabular}{|c|c|c|c|c|c|c|c|c|c|c|}
\hline \multirow{3}{*}{$\begin{array}{l}\text { Substrato }^{1} \\
\text { AA }\end{array}$} & \multicolumn{10}{|c|}{ Número de escleródios produzidos/isolados de Sclerotium rolfsii } \\
\hline & \multicolumn{2}{|c|}{ SR1 } & \multicolumn{2}{|c|}{ SR2 } & \multicolumn{2}{|c|}{ SR3 } & \multicolumn{2}{|c|}{ SR4 } & \multicolumn{2}{|c|}{ SR5 } \\
\hline & 5,6 & $\mathrm{D} b \mathrm{bc}$ & 69,3 & $\mathrm{C} \mathrm{a}$ & 1352,3 & $\mathrm{~B}$ a & 1,0 & $\mathrm{Dc}$ & 1611,0 & $\mathrm{~A} \mathrm{a}$ \\
\hline FA & 7,6 & $\mathrm{~B} \mathrm{~b}$ & 39,6 & $\mathrm{~B} a b$ & 1347,0 & A a & 49,6 & $\mathrm{~B}$ a & 1565,3 & A a \\
\hline QA & 1,6 & $\mathrm{D}$ cd & 26,6 & $\mathrm{C} \mathrm{b}$ & 659,3 & $\mathrm{Ab}$ & 2,0 & $\mathrm{D} b c$ & 540,0 & $\mathrm{~B} \mathrm{~b}$ \\
\hline SS & 0,0 & $\mathrm{Cd}$ & 32,3 & $\mathrm{~B} \mathrm{~b}$ & 597,0 & $\mathrm{~A} \mathrm{bc}$ & 10,6 & $\mathrm{~B} \mathrm{~b}$ & 619,6 & $\mathrm{Ab}$ \\
\hline $\mathrm{AB}$ & 0,0 & $\mathrm{Ad}$ & 0,0 & $\mathrm{Ac}$ & 0,0 & $\mathrm{Ad}$ & 0,0 & $\mathrm{Ac}$ & 0,0 & $\mathrm{Ac}$ \\
\hline $\mathrm{CA}$ & 104,0 & $\mathrm{~B} \mathrm{a}$ & 40,6 & $\mathrm{C} \mathrm{ab}$ & 464,0 & $\mathrm{Ac}$ & 0,0 & D c & 471,0 & $\mathrm{Ab}$ \\
\hline
\end{tabular}

* Média de quatro repetições. Médias seguidas pela mesma letra, maiúscula no sentido horizontal, e minúscula, no sentido vertical, não diferem entre si, a $5 \%$ de probabilidade pelo teste de Tukey. Dados transformados em $\sqrt{\mathrm{x}}+0,5$.

${ }^{1}$ AA - Aveia-ágar; FA - Fubá-ágar; QA - Quirera-ágar; SS - Sementes de sorgo; AB - Arroz beneficiado; CA - Casca de arroz. 


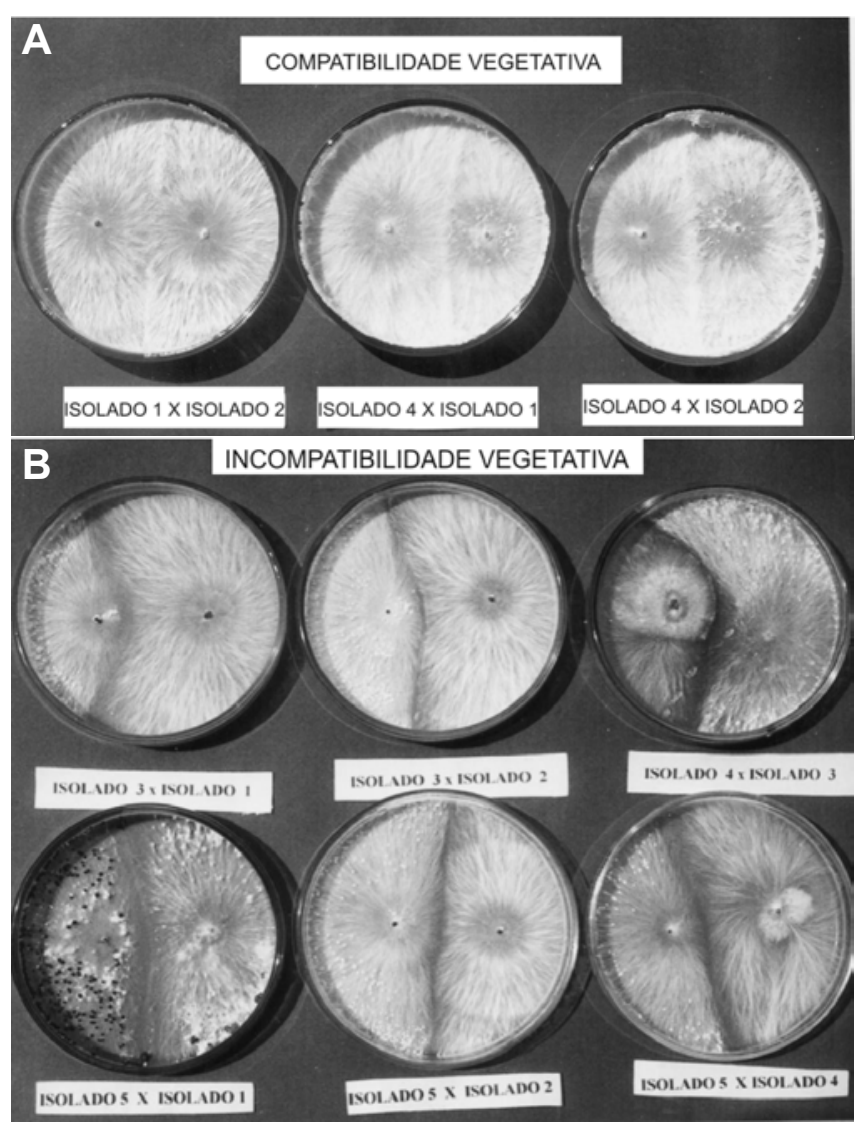

FIG. 1 - Pareamento de isolados de Sclerotium rolfsii mostrando reação de compatibilidade e incompatibilidade. (A) reação de compatibilidade entre os isolados SR1, SR2 e SR4 (B) reação de incompatibilidade entre SR3 e SR5 e os demais isolados.

de casca de arroz, como substrato para produção de escleródios de $S$. rolfsii. O baixo custo, bem como a fácil obtenção, tornam esse substrato com elevada potencialidade para a utilização nos estudos com este fungo.

Os isolados SR3 e SR5 apresentaram maior $(\mathrm{P} \leq 0,05)$ produção de escleródios, em quase todos os substratos estudados, destacando-se significativamente em aveia-ágar e fubá-ágar, sendo favorecidos pela luminosidade contínua (Tabelas 2 e 3). Os resultados sugerem a existência de diferenças genéticas quanto à formação de escleródios entre isolados, fato já observado anteriormente para este fungo por Ferraz \& Café Filho (1998); Harlton et al. (1995); Zoberi (1980). Deve ser destacado que estes mesmos isolados divergiram quanto à taxa crescimento micelial, apresentando SR3 e SR5 crescimento de $152,2 \mu \mathrm{m} / \mathrm{h}$ e $113,5 \mu \mathrm{m} / \mathrm{h}$, respectivamente, e quanto à compatibilidade vegetativa.

É importante salientar que os isolados SR3 e SR5, além de mostrar maior capacidade na produção de escleródios, cuja formação foi mais precoce (24-48 h) que os isolados SR1, SR2 e SR4, as colônias derivadas desses escleródios apresentaram menos vigor que aquelas oriundas de escleródios dos isolados SR1, SR2 e SR4. Estes últimos mostraram maior vigor na germinação, talvez em função do seu maior tamanho.
Observação similar foi verificada por Lang (1989) quando avaliou a produção de escleródios de dois isolados de $S$. rolfsii, obtidos de chuchu (Sechium edule Swartz) e feijoeiro (Phaseolus vulgaris L.), respectivamente.

Embora tenham sido utilizados poucos isolados, os resultados deste trabalho evidenciam que a variabilidade existente em $S$. rolfsii tem expressão distinta em função de características fisiológicas como crescimento micelial, compatibilidade vegetativa e produção de escleródios em diferentes substratos e regime de luminosidade.

\section{AGRADECIMENTOS}

Os autores agradecem ao Dr. Rildo Sartori B. Coelho (Universidade Federal Rural de Pernambuco) pela valiosa contribuição na revisão do manuscrito.

\section{REFERÊNCIAS BIBLIOGRÁFICAS}

AYCOCK, R. Stem rot and other diseases caused by Sclerotium rolfsii. North Caroline: Agricultural Experiment Station Tech. Bull, 174, 1966.

BEUTE, M.K. \& RODRIGUEZ-KABANA, R. Effects of volatile compounds from remoistened plant tissues on growth and germination of sclerotia of Sclerotium rolfsii. Phytopathology 69:802-805. 1979.

BLUM, L.E.B., PRADA, A., GALO, R. \& MEDEIROS, E. Efeito da temperatura, luz e meio de cultura na produção de escleródios de Sclerotium rolfsii e Sclerotinia sclerotiorum. Fitopatologia Brasileira 18:326. 1993. (Resumo).

CEASA-PE. Participação e procedência dos produtos comercializados na CEASA-PE. Central de Abastecimento de Pernambuco, RecifePE, 1992.

DANTAS, S.A.F. Métodos de inoculação de Sclerotium rolfsii Sacc., identificação de fontes de resistência em feijoeiro (Phaseolus vulgaris L.) e caracterização dos isolados do patógeno. (Tese de Mestrado). Recife. Universidade Federal Rural de Pernambuco. 1999.

EPPS, W.M., PATTERSON, J.C. \& FREEMAN, I.E. Physical, biological and chemical control integrated for soilborne disease in potatoes. Phytopathology 41:245-256. 1951.

FERNANDES, N.T., SANTOS, B.A., ZAMBOLIM, L., CHAVES, G.M. \& MIZUBUTI, E.S.G. Avaliação de meios de cultura naturais na produção de escleródios de Sclerotinia sclerotiorum (Lib.) de Bary. Fitopatologia Brasileira 18:323. 1993. (Resumo).

FERRAZ, L.C.L. \& CAFÉ FILHO, A.C. Meios de cultura e fatores culturais para a produção de escleródios e apotécios de Sclerotinia sclerotiorum in vitro. Fitopatologia Brasileira 23:364-369. 1998.

HARLTON, C.E., LÉVESQUE, C.A. \& PUNJA, Z.K. Genetic diversity in Sclerotium (Athelia) rolfsii and related species. Phytopathology 85:1269-1281. 1995.

HENIS, Y., CHET, I. \& AVIZHOHAR-HERSHENZON, Z. Nutricional and mechanical factory involved in mycelial growth and production of sclerotia by Sclerotium rolfsii in artificial medium and amended soil. Phytopathology 55:87-91. 1965.

KATAN, T., ZAMIR, D. \& SARFATTI, M. Vegetative compatibility in Fusarium oxysporum f.sp. radicis - lycopersici. Phytopathology 81:255-262. 1991. 
LANG, W. S. Caracterização cultural, morfológica e patogênica de Sclerotium rolfsii Sacc. e seu biocontrole por Trichoderma spp., "in vitro" e "in vivo". (Tese de Mestrado). Recife. Universidade Federal Rural de Pernambuco. 1989.

LESLIE, J.F. Fungal vegetative compatibility. Annual Review Phytopathology 31:127-150. 1993.

LILLY, V. G. \& BARNETT, H. L. Growth. Physiology of the fungi. New York, Mcgraw-Hill. 1951.

MATSUMOTO, M.N., HOMECHIN, M., MASSOLA, JR. N.S. \& KAMIKOGA, A.T.M. Efeito do substrato de cultivo na produção de escleródios e na patogenicidade de Sclerotium rolfsii. Summa Phytopathologica 26:91-94. 2000.

McCLELLAN, W.D., BORTHWICK, H.A., BJORNSSON, I. \& MARSHALL, B.H. Some responses of fungi to light. Phytopathology 45:465. 1955. (Abstract)

MENEZES, M. \& SILVA-HANLIN, D.M.W. Guia prático para fungos fitopatogênicos. Imprensa Universitária. UFRPE. Recife, 1997.

NAKATA, K. Studies on Sclerotium rolfsii Sacc. Part I. The phenomenon of aversion and its relation to the biologic forms of the fungus. Bulletin Science Fakult Terkult. 1:177-190. 1925.
NETO, J.V.P. \& BLUM, L.E.B. Avaliação do crescimento micelial e germinação de esclerócios de Sclerotium rolfsii em diferentes substratos. Fitopatologia Brasileira 29:637. 2004. (Resumo).

PÉREZ SENDÍN, M.A., GÓMEZ, I.G. \& GONZÁLEZ, B.J. Aspectos biológicos del hongo Sclerotium rolfsii en Cuba. Ciencias de la Agricultura 27:12-17. 1986.

PUNJA, Z.K. \& GROGAN, R.G. Basidiocarp induction, nuclear condition, variability, and heterokarion incompatibility in Athelia (Sclerotium rolfsii). Phytopathology 73:1273-1278. 1983.

PUNJA, Z K. \& JENKINS, S.F. Influence of temperature, moisture, modified gaseous atmosphere, and depth in soil on Sclerotium rolfsii. Phytopathology 74:749-754. 1984.

PUNJA, Z. K. The biology, ecology and control of Sclerotium rolfsii. Annual Review of Phytopathology 23:97-127. 1985.

PUNJA, Z.K. \& RAHE, J.E. Sclerotium. In: Singleton, L.L., Mihail, J.D. \& Rush, C.M. (Eds.). Methods for Research on Soilborne Phytopathogenic Fungi. St, Paul: APS Press. 1992. pp.166-170.

ZOBERI, M.H. Some nutritional factors regulating formation of sclerotial of Sclerotium rolfsii. Canadian Journal Botanic 58:24842490. 1980. 